\title{
Changes in sedentary behavior patterns during the transition from childhood to adolescence and their association with adiposity: a prospective study based on compositional data analysis
}

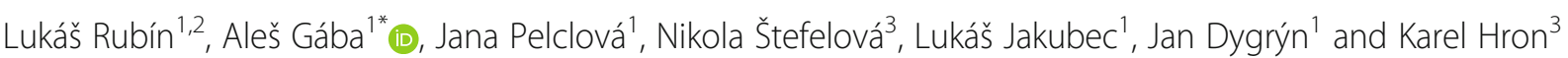

\begin{abstract}
Background: To date, no longitudinal study using a compositional approach has examined sedentary behavior (SB) patterns in relation to adiposity in the pediatric population. Therefore, our aims were to (1) investigate the changes in SB patterns and adiposity from childhood to adolescence, (2) analyze the prospective compositional associations between changes in SB patterns and adiposity, and (3) estimate the changes in adiposity associated with substituting SB with physical activity (PA) of different intensities.

Methods: The study presents a longitudinal design with a 5-year follow-up. A total of 88 participants (61\% girls) were included in the analysis. PA and SB were monitored for seven consecutive days using a hip-worn accelerometer. Adiposity markers (fat mass percentage [FM\%], fat mass index [FMI], and visceral adiposity tissue [VAT]) were assessed using the multi-frequency bioimpedance analysis. The prospective associations were examined using compositional data analysis.

Results: Over the follow-up period, the proportion of time spent in total SB increased by $154.8 \mathrm{~min} /$ day $(p<0.001)$. The increase in total SB was caused mainly by an increase in middle and long sedentary bouts, as these SB periods increased by $79.8 \mathrm{~min} /$ day and $62.0 \mathrm{~min} /$ day ( $p<0.001 \mathrm{for}$ both), respectively. FM\%, FMI, and VAT increased by $2.4 \%$ points, $1.0 \mathrm{~kg} / \mathrm{m}^{2}$, and $31.5 \mathrm{~cm}^{2}$ ( $p<0.001$ for all), respectively. Relative to the remaining movement behaviors, the increase in time spent in middle sedentary bouts was significantly associated with higher FM\% $\left(\beta_{\text {irr }}=0.27,95 \%\right.$ confidence interval [Cl]: 0.02 to 0.53 ) at follow-up. Lower VAT by 3.3\% (95\% Cl: 0.8 to 5.7), 3.8\% (95\% Cl: 0.03 to 7.4), 3.9\% (95\% Cl: 0.8 to 6.9), and 3.8\% (95\% Cl: 0.7 to 6.9) was associated with substituting 15 min/week spent in total SB and in short, middle, and long sedentary bouts, respectively, with an equivalent amount of time spent in vigorous PA.
\end{abstract}

\footnotetext{
* Correspondence: ales.gaba@upol.cz

${ }^{1}$ Faculty of Physical Culture, Palacký University Olomouc, Olomouc, Czech Republic

Full list of author information is available at the end of the article
}

(c) The Author(s). 2022 Open Access This article is licensed under a Creative Commons Attribution 4.0 International License, which permits use, sharing, adaptation, distribution and reproduction in any medium or format, as long as you give appropriate credit to the original author(s) and the source, provide a link to the Creative Commons licence, and indicate if changes were made. The images or other third party material in this article are included in the article's Creative Commons licence, unless indicated otherwise in a credit line to the material. If material is not included in the article's Creative Commons licence and your intended use is not permitted by statutory regulation or exceeds the permitted use, you will need to obtain permission directly from the copyright holder. To view a copy of this licence, visit http://creativecommons.org/licenses/by/4.0/. The Creative Commons Public Domain Dedication waiver (http://creativecommons.org/publicdomain/zero/1.0/) applies to the data made available in this article, unless otherwise stated in a credit line to the data. 
Conclusions: This study showed unfavorable changes in SB patterns and adiposity status in the transition from childhood to adolescence. Incorporating high-intensity PA at the expense of SB appears to be an appropriate approach to reduce the risk of excess adiposity in the pediatric population.

Keywords: Movement behaviors, Physical activity, Sedentary lifestyle, Prolonged sitting, Fat mass, Fat mass index, Visceral adipose tissue, Child, Adolescent

\section{Background}

The growing percentage of children who are classified as overweight or obese has reached an epidemic level worldwide and is considered one of the most serious public health challenges [1]. The number of children with an unhealthy weight has more than quadrupled in the past four decades [2], which has resulted in the current estimate of approximately one-fifth of youth being overweight or obese [3]. Scientific evidence has shown that overweight and obesity tend to trend upward from childhood to adolescence $[4,5]$ and, by extension, until adulthood [5, 6]. Excess adiposity leads to the development of several non-communicable diseases across the lifespan $[7,8]$ and has serious consequences such as premature mortality and physical morbidity in adulthood [9].

An increased accumulation of body fat is closely related to the way children spend their daily time. Previous studies have shown that unhealthy time-use characterized by an excessive amount of time spent in sedentary behavior ( $\mathrm{SB}$ ) at the expense of physical activity (PA) is associated with excess adiposity [10-12]. SB is a complex movement behavior of low energy expenditure $(\leq 1.5$ metabolic equivalents) and includes various behaviors occurring in different body postures (i.e., sitting, reclining, and lying down) [13]. In addition to total sedentary time, adiposity status is also associated with patterns of how SB accumulates. Since unfavorable adiposity status is associated with prolonged uninterrupted SB rather than with total SB [14], patterns of accumulation should be considered when investigating the role of $\mathrm{SB}$ in obesity prevention.

SB is considered a predominant component of a 24-h daily cycle. Therefore, compositional data analysis (CoDA) is recommended to find the optimal time-use for obesity prevention [15-17]. This analytical approach also allows the estimation of a theoretical change in adiposity resulting from substituting $\mathrm{SB}$ with other daily movement behaviors. Previous CoDA-based studies suggested that an effective strategy to control and prevent childhood obesity is to increase moderate-to-vigorous physical activity (MVPA) at the expense of total and prolonged SB $[10,14,18]$ and that adiposity status could also be improved by substituting longer sedentary bouts with shorter sedentary bouts [14]. Such estimates provide a guide for developing intervention strategies for obesity prevention and help existing interventions scale up their effectiveness.

To deepen the understanding of the role of SB in the prevention of excess adiposity, it appears important to describe changes in SB patterns during the transition from childhood to adolescence. It has been documented that an increase in total SB in this life period is caused primarily by prolonged uninterrupted SB $[19,20]$. Such age-related changes in daily time use may detrimentally influence adiposity when children grow older. However, evidence suggesting an optimal change in SB patterns for healthy adiposity during the transition from childhood to adolescence is lacking. To our knowledge, no CoDA-based study has estimated the effect of longitudinal changes in SB patterns in the pediatric population on adiposity status. Therefore, the main objectives of this study were to (1) investigate the longitudinal changes in SB patterns and adiposity markers from childhood to adolescence, (2) analyze the prospective compositional associations between changes in SB patterns from childhood to adolescence and adiposity, and (3) estimate the changes in adiposity associated with substituting SB with PA of different intensities.

\section{Methods}

\section{Participants}

Study participants were pupils from public elementary schools located in the eastern part of the Czech Republic (Moravia region). A total of 24 elementary schools were asked to participate in the study, 8 of which agreed. Sport schools (academies) or schools with special educational needs were not included. Data were intentionally collected in the similar spring and fall seasons from April 2013 to May 2014 for the baseline period and from April 2018 to May 2019 for the follow-up period to avoid any inconsistencies in weather conditions that could affect the movement behaviors of the participants.

The main inclusion criterion for study participants was good health as reported by parents or guardians. Participants with medical conditions that could affect engagement in habitual PA and/or adiposity were not included in the study. The study objectives and content of the research study were presented to the parents or guardians using an information booklet. The parents or guardians were also given a telephone number to inquire about any additional information or to clarify the 
objective and extent of the study. A total of 311 children participated in the study at baseline. Out of these, 88 participants $(61 \%$ girls) provided complete data for all variables of interest and were included in the final sample. A total of 223 participants were excluded from the study for various reasons (e.g., provided incomplete data, withdrawal). The final sample size was sufficient to ensure statistical power of $>80 \%$ in the regression model with 9 explanatory variables for alpha error of 0.05 and assuming at least medium effect size $\left(f^{2} \geq 0.2\right)$ in the population, according to Cohen [21]. The characteristics of the final sample are presented in Table 1.

Anthropometric measurements and adiposity assessment Each participant's standing height was measured before the adiposity assessment using a standardized procedure via an anthropometer P-375 (Trystom, Olomouc, Czech Republic) with an accuracy of $0.1 \mathrm{~cm}$. The body weight was measured to the nearest $0.1 \mathrm{~kg}$ via the device InBody 720 (InBody, Seoul, Korea). Sex- and age-standardized World Health Organization (WHO) body nass index (BMI) $z$-scores were calculated to categorize participants according to weight status [22].

The assessment of adiposity was performed by multifrequency $(1-1000 \mathrm{kHz})$ bioimpedance analysis using an InBody 720 body composition analyzer, which is considered highly precise for measuring body composition in the pediatric population [23]. Following the manufacturer's guidelines, the measurement was performed in a standing position, barefoot, and in light indoor clothing. The participants were instructed to fast for at least $4 \mathrm{~h}$ and maintain proper hydration for at least $24 \mathrm{~h}$ prior to

Table 1 Characteristics of participants at baseline and follow-up $(n=88)$

\begin{tabular}{|c|c|c|c|c|c|c|c|}
\hline & \multicolumn{2}{|c|}{ Baseline } & \multicolumn{2}{|c|}{ Follow-up } & \multicolumn{3}{|c|}{ Difference } \\
\hline & Mean $^{a}$ & $S D^{b}$ & Mean $^{a}$ & $S D^{b}$ & Mean $^{a}$ & $S D^{b}$ & $p$-value ${ }^{c}$ \\
\hline Age (years) & 9.2 & 0.9 & 14.6 & 0.8 & 5.4 & 0.3 & $<0.001$ \\
\hline Height (cm) & 137.4 & 7.3 & 166.4 & 8.4 & 29.0 & 5.1 & $<0.001$ \\
\hline Weight (kg) & 32.3 & 7.4 & 56.6 & 11.5 & 24.3 & 6.6 & $<0.001$ \\
\hline BMI z-score & 0.18 & 1.19 & 0.04 & 1.07 & -0.14 & 0.69 & 0.074 \\
\hline Fat mass (\%) & 16.2 & 8.3 & 18.6 & 8.6 & 2.4 & 6.1 & $<0.001$ \\
\hline Fat mass index $\left(\mathrm{kg} / \mathrm{m}^{2}\right)$ & 2.9 & 2.1 & 4.0 & 2.4 & 1.1 & 1.6 & $<0.001$ \\
\hline Visceral adipose tissue $\left(\mathrm{cm}^{2}\right)$ & 27.4 & 23.4 & 45.3 & 31.5 & 17.9 & 21.8 & $<0.001$ \\
\hline \multicolumn{8}{|l|}{ Weight status $^{d}$} \\
\hline Underweight (\%) & 2.3 & & 1.1 & & -1.2 & & 1.000 \\
\hline Normal weight (\%) & 73.8 & & 76.1 & & 2.3 & & 0.803 \\
\hline Overweight (\%) & 14.8 & & 20.5 & & 5.7 & & 0.359 \\
\hline Obesity (\%) & 9.1 & & 2.3 & & -6.8 & & 0.041 \\
\hline \multicolumn{8}{|c|}{ Movement behaviors (the 4-part composition) } \\
\hline Total SB (min/day) & 328.2 & 49.8 & 483.0 & 44.6 & 154.8 & 41.5 & $<0.001$ \\
\hline LPA (min/day) & 354.3 & 42.3 & 260.6 & 42.0 & -93.7 & 39.4 & $<0.001$ \\
\hline MPA (min/day) & 41.1 & 42.4 & 28.6 & 38.0 & -12.5 & 38.1 & $<0.001$ \\
\hline VPA (min/day) & 12.8 & 65.5 & 16.2 & 75.5 & 3.4 & 81.0 & 0.005 \\
\hline \multicolumn{8}{|c|}{ Movement behaviors (the 6-part composition) } \\
\hline SB short bouts (min/day) & 195.4 & 22.9 & 209.7 & 22.7 & 14.3 & 22.8 & $<0.001$ \\
\hline SB middle bouts ( $\mathrm{min} /$ day) & 101.5 & 28.6 & 181.3 & 24.4 & 79.8 & 27.7 & $<0.001$ \\
\hline SB long bouts (min/day) & 36.5 & 47.9 & 98.5 & 42.8 & 62.0 & 45.4 & $<0.001$ \\
\hline LPA (min/day) & 352.1 & 23.1 & 255.9 & 24.2 & -96.2 & 24.7 & $<0.001$ \\
\hline MPA (min/day) & 39.6 & 27.6 & 28.2 & 26.7 & -11.4 & 27.1 & $<0.001$ \\
\hline VPA (min/day) & 11.3 & 49.9 & 14.8 & 59.2 & 3.5 & 52.3 & 0.868 \\
\hline
\end{tabular}

Bold values denote significant results

LPA light physical activity, MPA moderate physical activity, SB sedentary behavior, SD standard deviation, VPA vigorous physical activity

${ }^{a}$ The robust mean for compositional data; arithmetic mean for non-compositional continuous variables; percentages for categorical variables

${ }^{b}$ Part of the total variance related to a given time-use component for compositional data; standard deviation for other variables

${ }^{c}$ Analyzed using the paired sample t-test for continuous variables (the first pivot coordinate was used to represent each time-use variable for compositional data) and the chi-squared test for categorical variables

${ }^{\mathrm{d}}$ Based on BMI z-score categories 
the examination to maintain the validity of the measurement. One field worker performed the measurement on the school premises. Adiposity was expressed as the fat mass percentage (FM\%, \%), fat mass index (FMI, $\mathrm{kg} / \mathrm{m}^{2}$ ), and visceral adiposity tissue (VAT, $\mathrm{cm}^{2}$ ).

\section{Physical activity and sedentary behavior assessment}

A hip-mounted ActiGraph GT3X accelerometer (ActiGraph, Pensacola, FL, USA) was used to assess PA and SB for seven consecutive days. A full description of the measurement protocol is provided elsewhere [24]. Briefly, participants were instructed to wear the device during the waking time period each day excluding time when they were in contact with water (e.g., bathing or swimming). Before monitoring, the accelerometers were initialized to collect data in $1 \mathrm{~min}$ intervals. Non-wear time was defined by an interval of at least 60 consecutive minutes of zero activity intensity counts [25], not allowing any interruptions [26]. For wear time data, SB was defined as 0-100 counts per minute (cpm), light PA (LPA) as 101-2295 cpm, moderate PA (MPA) as 2296$4011 \mathrm{cpm}$, and vigorous PA (VPA) as $\geq 4012 \mathrm{cpm}$ [27]. The Evenson cut-off points provided an acceptable classification accuracy for all levels of PA intensity among children and adolescents [28]. In addition, these cut-off points are the most common cut-off points used in studies evaluating longitudinal changes in PA in children and adolescents [29]. A sedentary bout was defined as 1 or more consecutive minutes with fewer than $100 \mathrm{cpm}$. Sedentary bouts with durations of 1-9 min, 10-29 min, and $\geq 30 \mathrm{~min}$, that is, short, middle, and long sedentary bouts, respectively, were analyzed. The thresholds for sedentary bouts were consulted based on the previous literature [30] and in order to minimize the number of zero values. Accelerometer data were considered valid if the participant wore the device for at least 4 days, including 1 weekend day, with $\geq 10 \mathrm{~h}$ of wear time per school day and $\geq 8$ h of wear time per weekend day [31]. Only those participants who had valid accelerometer data for both baseline and follow-up were acceptable for analysis.

\section{Statistical analyses}

Statistical analyses were conducted using IBM SPSS Statistics version 23 (IBM, Armonk, NY, USA) and R version 3.4.2 ( $\mathrm{R}$ Foundation for Statistical Computing, Vienna, Austria). The differences between baseline and follow-up were analyzed using the paired sample $t$-test. The chi-squared test was used to compare proportions. One-way analysis of covariance adjusted for age and sex was used to perform a sensitivity analysis to assess differences between the participants included and excluded from the study.
CoDA was carried out to analyze differences in the time-use patterns between the baseline and follow-up movement behavioral data. The robust compositional mean and the variation matrix were calculated for the purpose of descriptive statistics [32] to reduce the influence of possible aberrant observations. To analyze the data, two wake-time compositions were created. The 4-part composition consisted of total SB, LPA, MPA, and VPA, and the 6-part composition consisted of total SB decomposed into bouts of different durations (i.e., short, middle, and long sedentary bouts), LPA, MPA, and VPA. Accordingly, the relative information about the other components (LPA, MPA and VPA) also necessarily changed between the compositions, as each of these components was determined by five log-ratios in the latter compostion instead of three in the former composition. A Bayesian-multiplicative approach [33] was used to replace zero values that occurred in long sedentary bouts in $3 \%$ of participants. The compositions were expressed in the pivot coordinates system, being a special case of isometric log-ratios (ilrs). From each of the coordinate systems assessed, only the first pivot coordinate (i.e., $i l r_{1}$ ) was interpreted in all analyses.

A robust compositional regression analysis [32] was performed to analyze the prospective associations between the changes in SB patterns and adiposity. Based on our previous experience [34], we used regression models in which the differences between follow-up and baseline SB patterns (in terms of pivot coordinates) were set as the independent variables, and adiposity markers at follow-up were set as the dependent variables. The regression models were further adjusted for the corresponding dependent variables at baseline and the sex and age of the participants. Isotemporal substitution modeling was used to estimate the changes in different adiposity markers associated with one-to-one reallocation between SB and PA. For this purpose, wake-time compositions were closed to $16 \mathrm{~h}$ because of the assumption of an average of $8 \mathrm{~h}$ of sleep per day based on previous reports of sleep duration in the target population [35]. Estimated changes in adiposity markers were considered significant when $95 \%$ confidence intervals (CIs) did not cover zero. All statistical analyses were conducted at a significance level of $p<0.05$.

\section{Results}

A total of 311 participants with a mean age of $9.2 \pm 1.0$ years participated in the study at baseline. Of these, 135 participants attended the follow-up investigation. Of all participants who attended the follow-up investigation, 88 provided complete data for all variables of interest and were included in the final analysis. No significant differences in adiposity indicators were observed between participants who were included and excluded 
(Table S1). Participants who were included only had lower body height $(p<0.001)$ and weight $(p=0.006)$ than those who were excluded.

The characteristics of the study participants included in the final analysis are presented in Table 1. For the final sample, the mean follow-up duration was $5.0 \pm 0.1$ years. The median number of valid days was 7 days for both time points. Nearly $24 \%$ of participants were overweight and obese at baseline, and FM\%, FMI, and VAT increased by $2.4 \%$ points, $1.1 \mathrm{~kg} / \mathrm{m}^{2}$, and $17.9 \mathrm{~cm}^{2}$ $(p<0.001$ for all), respectively, over the 5 -year period. The proportion of time spent in all movement behaviors within the 4-part waking-time composition changed significantly over the follow-up period. Specifically, the proportion of time spent in total SB and VPA increased by $47 \%$ (difference: $154.8 \mathrm{~min} /$ day; $p<0.001$ ) and $27 \%$ (difference: $3.4 \mathrm{~min} /$ day; $p=0.005$ ), respectively, while the proportions of time spent in LPA and MPA decreased by $26 \%$ (difference: $93.7 \mathrm{~min} /$ day; $p<0.001$ ) and $30 \%$ (difference: $12.5 \mathrm{~min} /$ day; $p<0.001$ ), respectively. The increase in total SB was caused mainly by an increase in the middle and long sedentary bouts, as they increased by $79 \%$ (difference: $79.8 \mathrm{~min} /$ day; $p<0.001$ ) and $170 \%$ (difference: $62.0 \mathrm{~min} /$ day; $p<0.001$ ), respectively.

The prospective compositional associations between the changes in movement behaviors and adiposity indicators are displayed in Table 2. Relative to the remaining movement behaviors, the increase in time spent in middle sedentary bouts was significantly associated with higher FM\% $\left(\beta_{\text {ilr } 1}=0.27,95 \%\right.$ CI: 0.02 to 0.53$)$ at follow-up. Moreover, the increase in time spent in VPA was associated with lower VAT within the 4-part $\left(\beta_{\mathrm{ilr} 1}=-0.30,95 \% \mathrm{CI}:-0.52\right.$ to -0.08$)$ and 6-part $\left(\beta_{\mathrm{ilr} 1}=-0.30,95 \% \mathrm{CI}:-0.54\right.$ to -0.05$)$ wakingtime compositions. The estimated changes in the adiposity indicators associated with time reallocation between SB and PA of different intensities are presented in Table 3 and Fig. 1. Favorable adiposity status was associated with substituting SB with PA only for VAT. Specifically, substituting $15 \mathrm{~min}$ of total SB or short, middle, and long sedentary bouts with an equivalent amount of VPA was associated with a reduction in VAT of $3.3 \%$ (95\% CI: 0.8 to 5.7 ), $3.8 \%$ (95\% CI: 0.03 to 7.4 ), $3.9 \%$ (95\% CI: 0.8 to 6.9 ), and 3.8 (95\% CI: 0.7 to 6.8 ), respectively.

\section{Discussion}

Changes in adiposity and movement behaviors were revealed during the transition from childhood to adolescence. All adiposity markers, total SB, and VPA increased, while LPA and MVPA decreased. A compositional prospective association between the changes in the proportion of time spent in middle sedentary bouts and FM\% in adolescence was found. Moreover, a change in VPA was associated with VAT in adolescence. Favorable changes in adiposity status were identified when the amounts of time spent in total SB and sedentary bouts of different durations were substituted with VPA.

Our findings on the age-related changes in adiposity and movement behaviors are in accordance with the available evidence [36-38]. We found that the increase in the proportion of time spent in total $\mathrm{SB}$ was

Table 2 Compositional robust regression model estimates for the adiposity markers of 5-year changes

\begin{tabular}{|c|c|c|c|c|c|c|c|c|c|}
\hline & \multicolumn{3}{|c|}{$\begin{array}{l}\text { Fat mass } \\
(\%)\end{array}$} & \multicolumn{3}{|c|}{$\begin{array}{l}\text { Fat mass index } \\
\left(\mathrm{kg} / \mathrm{m}^{2}\right)\end{array}$} & \multicolumn{3}{|c|}{$\begin{array}{l}\text { Visceral adipose tissue } \\
\left(\mathrm{cm}^{2}\right)\end{array}$} \\
\hline & $\beta_{\text {ilr1 }}$ & $95 \% \mathrm{Cl}$ & $p$-value & $\beta_{\mathrm{ilr} 1}$ & $95 \% \mathrm{Cl}$ & $p$-value & $\beta_{\text {ilr1 }}$ & $95 \% \mathrm{Cl}$ & $p$-value \\
\hline \multicolumn{10}{|c|}{ Model based on the 4-part composition } \\
\hline SB (h/week) & 0.06 & $(-0.30,0.41)$ & 0.757 & 0.03 & $(-0.39,0.45)$ & 0.888 & 0.48 & $(-0.27,1.23)$ & 0.209 \\
\hline LPA (h/week) & 0.00 & $(-0.41,0.41)$ & 0.990 & 0.03 & $(-0.46,0.52)$ & 0.890 & -0.25 & $(-1.09,0.59)$ & 0.561 \\
\hline MPA (h/week) & 0.00 & $(-0.18,0.19)$ & 0.977 & 0.01 & $(-0.22,0.25)$ & 0.910 & 0.07 & $(-0.34,0.48)$ & 0.735 \\
\hline VPA (h/week) & -0.06 & $(-0.13,0.02)$ & 0.157 & -0.07 & $(-0.17,0.02)$ & 0.141 & -0.30 & $(-0.52,-0.08)$ & 0.010 \\
\hline \multicolumn{10}{|c|}{ Model based on the 6-part composition } \\
\hline SB short bouts (h/week) & -0.38 & $(-0.87,0.12)$ & 0.132 & -0.25 & $(-0.82,0.33)$ & 0.400 & 0.25 & $(-1.01,1.51)$ & 0.693 \\
\hline SB middle bouts (h/week) & 0.27 & $(0.02,0.53)$ & 0.034 & 0.24 & $(-0.09,0.58)$ & 0.152 & 0.22 & $(-0.39,0.82)$ & 0.482 \\
\hline SB long bouts (h/week) & -0.04 & $(-0.14,0.07)$ & 0.496 & -0.05 & $(-0.17,0.06)$ & 0.357 & 0.06 & $(-0.12,0.24)$ & 0.499 \\
\hline LPA (h/week) & 0.19 & $(-0.28,0.66)$ & 0.422 & 0.10 & $(-0.54,0.73)$ & 0.766 & -0.32 & $(-1.61,0.97)$ & 0.620 \\
\hline MPA (h/week) & -0.01 & $(-0.18,0.16)$ & 0.879 & 0.03 & $(-0.20,0.26)$ & 0.824 & 0.09 & $(-0.35,0.53)$ & 0.684 \\
\hline VPA (h/week) & -0.04 & $(-0.11,0.03)$ & 0.278 & -0.07 & $(-0.17,0.03)$ & 0.187 & -0.30 & $(-0.54,-0.05)$ & 0.019 \\
\hline
\end{tabular}

Independent variables are expressed as the first pivot coordinate which represents the relative contribution of one behavior relative to remaining behaviors All regression models were adjusted for age, sex, and fat mass percentage at baseline Bold values denote significant results

Cl confidence interval, ilr1 isometric log-ratio (the first pivot coordinate), LPA light physical activity, MPA moderate physical activity, SB sedentary behavior, VPA vigorous physical activity 
Table 3 Estimated changes in adiposity markers associated with isotemporal substitutions between sedentary behavior and light, moderate, and vigorous physical activity

\begin{tabular}{|c|c|c|c|c|c|c|}
\hline & \multicolumn{2}{|c|}{$\begin{array}{l}2 \mathrm{~h} / \text { week } \\
\text { from SB to LPA }\end{array}$} & \multicolumn{2}{|c|}{$\begin{array}{l}1 \mathrm{~h} / \text { week } \\
\text { from SB to MPA }\end{array}$} & \multicolumn{2}{|c|}{$\begin{array}{l}15 \mathrm{~min} / \text { week } \\
\text { from SB to VPA }\end{array}$} \\
\hline & $\begin{array}{l}\text { Percentage } \\
\text { change }\end{array}$ & $95 \% \mathrm{Cl}$ & $\begin{array}{l}\text { Percentage } \\
\text { change }\end{array}$ & $95 \% \mathrm{Cl}$ & $\begin{array}{l}\text { Percentage } \\
\text { change }\end{array}$ & $95 \% \mathrm{Cl}$ \\
\hline \multicolumn{7}{|l|}{ Fat mass (\%) } \\
\hline Total SB & -0.2 & $(-2.7,2.3)$ & -0.1 & $(-2.3,2.2)$ & -0.6 & $(-1.5,0.3)$ \\
\hline SB short bouts & 3.1 & $(-1.5,7.9)$ & 1.0 & $(-1.2,3.3)$ & -0.2 & $(-1.4,1.0)$ \\
\hline SB middle bouts & -2.8 & $(-6.1,0.7)$ & -1.8 & $(-4.7,1.1)$ & -0.9 & $(-1.8,0.01)$ \\
\hline SB long bouts & 2.2 & $(-2.7,7.2)$ & 0.5 & $(-2.7,3.7)$ & -0.3 & $(-1.3,0.6)$ \\
\hline \multicolumn{7}{|c|}{ Fat mass index $\left(\mathrm{kg} / \mathrm{m}^{2}\right)$} \\
\hline Total SB & 0.0 & $(-3.0,3.0)$ & 0.2 & $(-2.6,3.0)$ & -0.8 & $(-1.8,0.3)$ \\
\hline SB short bouts & 1.9 & $(-3.6,7.7)$ & 1.1 & $(-1.9,4.3)$ & -0.6 & $(-2.1,0.9)$ \\
\hline SB middle bouts & -2.7 & $(-7.6,2.4)$ & -1.1 & $(-4.6,2.5)$ & -1.2 & $(-2.5,0.2)$ \\
\hline SB long bouts & 2.5 & $(-2.8,8.0)$ & 1.3 & $(-2.6,5.4)$ & -0.6 & $(-1.9,0.7)$ \\
\hline \multicolumn{7}{|c|}{ Visceral adipose tissue $\left(\mathrm{cm}^{2}\right)$} \\
\hline Total SB & -2.5 & $(-7.4,2.7)$ & 0.1 & $(-4.7,5.1)$ & -3.3 & $(-5.7,-0.8)$ \\
\hline SB short bouts & -2.6 & $(-13.6,9.8)$ & 0.5 & $(-4.0,5.2)$ & -3.8 & $(-7.4,-0.03)$ \\
\hline SB middle bouts & -3.7 & $(-12.0,5.3)$ & -0.1 & $(-7.3,7.7)$ & -3.9 & $(-6.9,-0.8)$ \\
\hline SB long bouts & -3.5 & $(-11.6,5.3)$ & 0.2 & $(-6.5,7.3)$ & -3.8 & $(-6.8,-0.7)$ \\
\hline
\end{tabular}

Bold values denote significant changes in adiposity status

Cl confidence interval, LPA light physical activity, MPA moderate physical activity, SB sedentary behavior, VPA vigorous physical activity

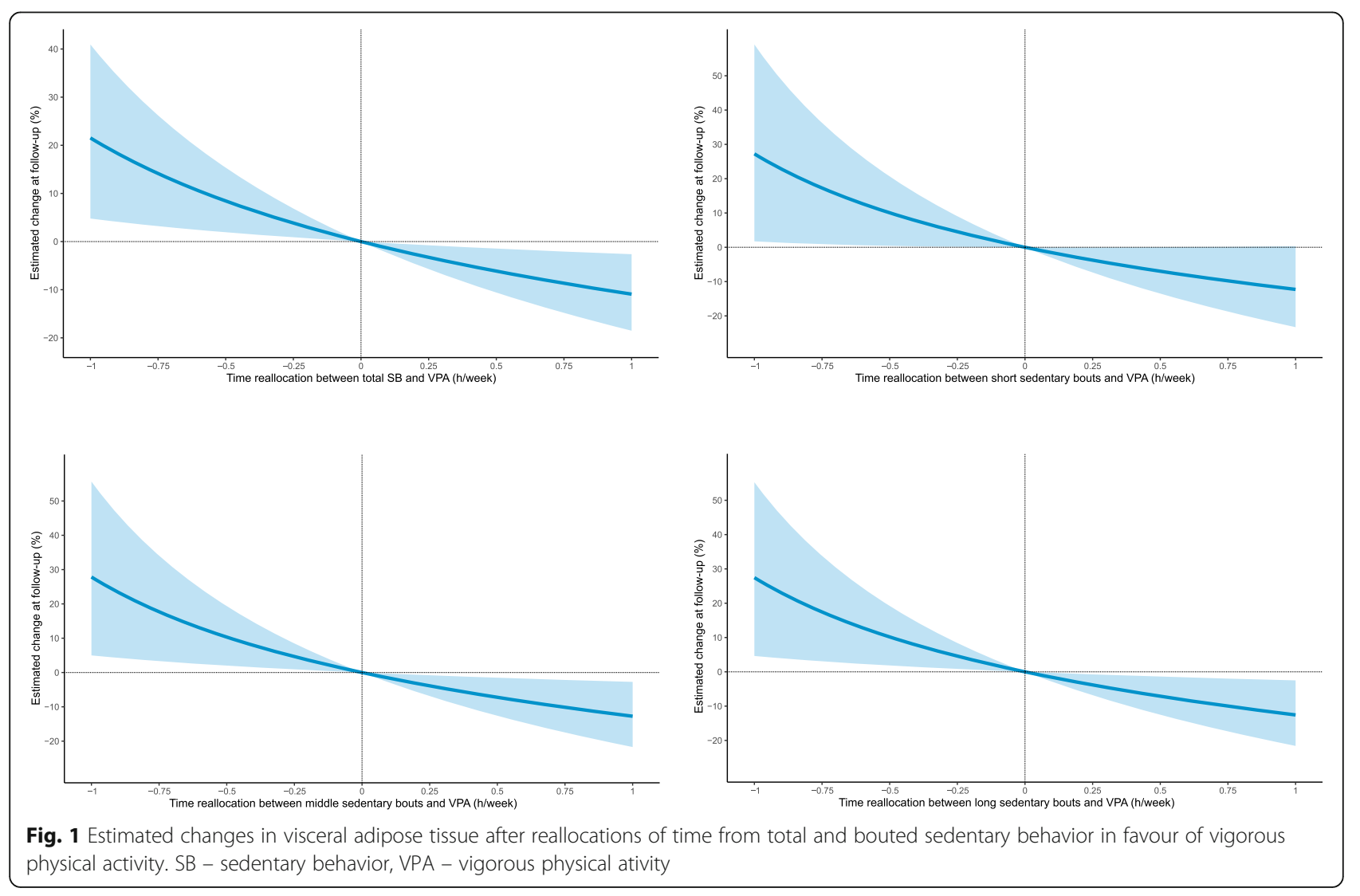


predominantly caused by an increase in the time spent engaging in prolonged SB. Similar results were observed in 5991 participants included in a pooled analysis of longitudinal data from the International Children's Accelerometry Database [20]. Moreover, the proportion of time spent in middle and long sedentary bouts increased at the expense of LPA and MPA in the current study. This implies that SB becomes less fragmented, as it is probably less interrupted by brief bouts of PA. This assumption might be supported by the longitudinal analysis performed by Janssen and colleagues [19], who found a decrease in the frequency of sedentary breaks and an increase in the sedentary bout duration across childhood and adolescence.

One possible explanation for the decrease in sedentary fragmentation could be a change in movement behaviors associated with the transition to secondary school [39]. As most school time corresponds to SB [40, 41] and considering the SB patterns during lessons (i.e., prolonged uninterrupted sitting), it can be assumed that the increase in prolonged SB is caused mainly by an increase in school-based SB. Another contributor to prolonged SB could be the increased time spent traveling to school in a car or bus. Adolescents are probably more likely to commute to schools located farther away from their place of residence compared with children [42]. Likewise, an age-related decrease in non-organized leisure time activities may contribute to the prolonged SB across childhood and adolescence [43]. If further longitudinal studies confirm these assumptions, interventions targeting the interruption of prolonged sitting during school and leisure time should be implemented into public health strategies.

The change in SB patterns during the transition from childhood to adolescence could have several health implications. According to this study, an increase in the proportion of middle sedentary bouts of the 10-29 min duration (relative to the remaining movement behaviors) is prospectively associated with adiposity in adolescence. Similarly to our findings, Mann and colleagues [44] showed that an increase in sedentary fragmentation expressed as an increase of one bout per sedentary hour per year was significantly associated with a decrease in BMI and FMI between 9 and 12 years of age. It seems that breaking down prolonged uninterrupted sedentary periods by sporadic PA might be an appropriate strategy for optimizing movement behaviors in the pediatric population. However, the ability to compare our findings with those previously published is limited, as, to the best our knowledge, the current study is the first to investigate prospective associations between SB patterns and adiposity using the CoDA approach. Despite this limitation, it appears that changes in SB patterns play an important role in the accumulation of adipose tissue in adolescence. Future studies are needed to identify the potential determinants of SB pattern changes, as this was beyond the scope of the present study.

We emphasize that an increase in time spent in VPA is associated with lower central adiposity in adolescence. This is in accordance with the review by Gralla and colleagues [45], who suggested that VPA is a stronger predictor for central adiposity than other intensities of PA. In light of this finding, we suggest one possible strategy to reduce adiposity is interrupting prolonged SB by bouts of VPA. This hypothesis is supported by our results from the isotemporal substitution analysis, which indicate an improved adiposity status when $15 \mathrm{~min}$ of total SB or all sedentary bouts are reallocated in favor of VPA. However, it is questionable whether that change could be sustainable, as it would mean more than doubling the amount of time spent in VPA in our sample. An alternative strategy may be interrupting SB by very short bouts (i.e., < $1 \mathrm{~min}$ ) of VPA. This specific point could not be addressed in the current study, as the movement behaviors were assessed using a $60 \mathrm{~s}$ sampling interval. However, the findings from an experimental study conducted in children with overweight and obesity [46], for whom VPA may be difficult to achieve, support this strategy by showing an acute improvement in metabolic markers in response to interrupting SB with very short bouts of activity of lower intensity.

The current study has several strengths. First, this is the only study that considers the compositional nature of movement behavior data in analyzing the prospective associations between changes in SB patterns and adiposity. The utilization of the CoDA approach allows the control of regression models for all movement behaviors (i.e., using the set of the first pivot coordinates in the models) and the avoidance of biased estimates due to multicollinearity. Second, the device-based assessment of both adiposity markers and movement behaviors provides reliable and valid data. Finally, the SB patterns were analyzed as well, which allowed the accurate differentiation of prolonged SB from total SB.

There are also limitations within this study. Our findings should be interpreted with caution, as the study is limited to movement behaviors. Omitting time spent asleep may lead to biased estimates of regression analysis. Another source of bias relates to analyzing SB without distinguishing body postures (i.e., the differentiation of sedentary postures from standing). Thus, longitudinal CoDA-based studies based on a 24-h wear time protocol and the combination of intensity- and posturespecific assessments of movement behaviors are warranted to analyze the associations between SB patterns and adiposity across childhood and adolescence. Although the regression models were adjusted for several confounding factors, there are still potential endo- and 
exogenous determinants of adiposity that have not been considered. Since youth movement behaviors are characterized by their intermittent nature, the 1-min sampling interval used in this study could lead to the underestimation of high-intensity PA and may not capture all breaks in SB [47]. Another limitation is the relatively high loss of participants. However, a sensitivity analysis (Table S1) confirmed no significant differences in outcome variables between participants included and excluded from the study. Finally, our findings are not fully generalizable due to the small sample size and the higher proportion of girls. These limitations did not allow us to focus on the sex differences in associations between longitudinal changes in SB patterns and adiposity. Further research with larger samples is warranted to explore the longitudinal compositional associations separately for boys and girls.

\section{Conclusion}

The present study revealed age-related changes in adiposity markers and movement behaviors. The increase in prolonged SB at the expense of PA implies that unhealthy time-use patterns develop during the transition from childhood to adolescence. Relative to the remaining movement behaviors, an increase in the proportion of time spent in prolonged SB over this transition period was associated with greater adiposity in adolescence, while an increase in VPA was associated with reduced central adiposity. Substituting total SB with VPA appears to be an appropriate target for intervention strategies, as high-intensity PA was associated with favorable adiposity status. These findings may help design more effective interventions to prevent an unhealthy gain in adiposity during the transition from childhood to adolescence.

\section{Abbreviations \\ BMI: Body mass index; Cl: Confidence interval; CoDA: Compositional data analysis; cpm: Counts per minute; FM\%: Fat mass percentage; FMl: Fat mass index; ilr: Isometric log-ratio; LPA: Light physical activity; MPA: Moderate physical activity; MVPA: Moderate-to-vigorous physical activity; PA: Physical activity; SB: Sedentary behavior; VAT: Visceral adiposity tissue; VPA: Vigorous physical activity; WHO: World Health Organization}

\section{Supplementary Information}

The online version contains supplementary material available at https://doi. org/10.1186/s13690-021-00755-5.

Additional file 1: Table S1. Characteristics of included and excluded participants.

\section{Acknowledgements}

The authors are grateful to all the participants involved in this study.

\section{Authors' contributions}

$L R$ and $A G$ conceptualized and designed this study; $L R, L, J D$, and $A G$ collected the data; $L R, A G$, and JD prepared final dataset; $L R$, NŠ, $K H$, and $A G$ analyzed and interpreted the data; LR drafted the manuscript; AG provided intellectual role in improving the manuscript; AG and JP provided major roles in revising the manuscript. All authors read and approved the final manuscript.

\section{Funding}

This research was funded by research grants from the Czech Science Foundation (18-091885 and 22-02392S).

\section{Availability of data and materials}

The dataset analyzed during the current study is available in the Figshare repository (https://doi.org/10.6084/m9.figshare.14260541).

\section{Declarations}

Ethics approval and consent to participate

The study was approved under reference number 53/2012 on 18 December 2012 (updated on 16 March 2017 under reference number 19/2017) by the Ethics Committee of the Faculty of Physical Culture, Palacký University Olomouc, which is governed by the ethical standards set out in the World Medical Association Declaration of Helsinki and its later amendments. Prior to the implementation of the research the participants' parents or guardians signed a written informed consent form.

Consent for publication

Not applicable.

\section{Competing interests}

The authors declare that they have no competing interests.

\section{Author details}

${ }^{1}$ Faculty of Physical Culture, Palacký University Olomouc, Olomouc, Czech Republic. ${ }^{2}$ Faculty of Science, Humanities and Education, Technical University of Liberec, Liberec, Czech Republic. ${ }^{3}$ Faculty of Science, Palacký University Olomouc, Olomouc, Czech Republic.

Received: 21 September 2021 Accepted: 8 December 2021

Published online: 04 January 2022

\section{References}

1. World Health Organization. Obesity and overweight [Internet]. 2021. [cited 2021 Jan 3]. Available from: https://www.who.int/news-room/fact-sheets/ detail/obesity-and-overweight.

2. NCD Risk Factor Collaboration. Worldwide trends in body-mass index, underweight, overweight, and obesity from 1975 to 2016: a pooled analysis of 2416 population-based measurement studies in 128.9 million children, adolescents, and adults. Lancet. 2017:390(10113):2627-42.

3. Ng M, Fleming T, Robinson M, Thomson B, Graetz N, Margono C, et al. Global, regional, and national prevalence of overweight and obesity in children and adults during 1980-2013: a systematic analysis for the global burden of disease study 2013. Lancet. 2014:384(9945):766-81.

4. Ronque ERV, Werneck AO, Bueno MRO, Cyrino ES, Stanganelli LCR, Arruda M. Tracking of body adiposity indicators from childhood to adolescence: mediation by BMI. PLoS One. 2018;13(2):e0191908.

5. Araújo J, Severo M, Barros H, Mishra GD, Guimarães JT, Ramos E. Developmental trajectories of adiposity from birth until early adulthood and association with cardiometabolic risk factors. Int J Obes. 2015;39:1443-9.

6. Blasquez Shigaki G, Barbosa CCL, Batista MB, Romanzini CLP, Gonçalves EM, Serassuelo Junior $\mathrm{H}$, et al. Tracking of health-related physical fitness between childhood and adulthood. Am J Hum Biol. 2020;32(4):e23381.

7. Sahoo K, Sahoo B, Choudhury AK, Sofi NY, Kumar R, Bhadoria AS. Childhood obesity: causes and consequences. J Fam Med Prim Care. 2015;4(2):187-92.

8. Williams EP, Mesidor M, Winters K, Dubbert PM, Wyatt SB. Overweight and obesity: prevalence, consequences, and causes of a growing public health problem. Curr Obes Rep. 2015;4:363-70.

9. Reilly JJ, Kelly J. Long-term impact of overweight and obesity in childhood and adolescence on morbidity and premature mortality in adulthood: systematic review. Int J Obes. 2011;35(7):891-8.

10. Dumuid D, Stanford TE, Pedišić Ž, Maher C, Lewis LK, Martín-Fernádez J-A et al. Adiposity and the isotemporal substitution of physical activity, sedentary time and sleep among school-aged children: a compositional data analysis approach. BMC Public Health. 2018;18(1):311. 
11. Grgic J, Dumuid D, Bengoechea EG, Shrestha N, Bauman A, Olds T, et al. Health outcomes associated with reallocations of time between sleep, sedentary behaviour, and physical activity: a systematic scoping review of isotemporal substitution studies. Int J Behav Nutr Phys Act. 2018;15:69.

12. Dumuid D, Wake M, Burgner D, Tremblay MS, Okely AD, Edwards B, et al. Balancing time use for children's fitness and adiposity: evidence to inform 24-hour guidelines for sleep, sedentary time and physical activity. PLoS One. 2021:16(1):e0245501.

13. Tremblay MS, Aubert S, Barnes JD, Saunders TJ, Carson V, Latimer-Cheung AE, et al. Sedentary behavior research network (SBRN) - terminology consensus project process and outcome. Int J Behav Nutr Phys Act. 2017;14:75.

14. Gába A, Pedišić Ž, Štefelová N, Dygrýn J, Hron K, Dumuid D, et al. Sedentary behavior patterns and adiposity in children: a study based on compositional data analysis. BMC Pediatr. 2020;20:147.

15. Pedišić Ž, Dumuid D, Olds TS. Integrating sleep, sedentary behaviour, and physical activity research in the emerging field of time-use epidemiology: definitions, concepts, statistical methods, theoretical framework, and future directions. Kinesiology. 2017;49(2):252-69.

16. Dumuid D, Pedišić Ž, Palarea-Albaladejo J, Martín-Fernández JA, Hron K, Olds T. Compositional data analysis in time-use epidemiology: what, why, how. Int J Environ Res Public Health. 2020;17(7):2220.

17. Migueles JH, Aadland E, Andersen LB, Brønd JC, Chastin SF, Hansen BH, et al. GRANADA consensus on analytical approaches to assess associations with accelerometer-determined physical behaviours (physical activity, sedentary behaviour and sleep) in epidemiological studies. Br J Sports Med. 2021. https:// bjsm.bmj.com/content/early/2021/04/14/bjsports-2020-103604.info.

18. Dumuid D, Wake M, Clifford S, Burgner D, Carlin JB, Mensah FK, et al. The association of the body composition of children with 24-hour activity composition. J Pediatr. 2019;208:43-49.e9.

19. Janssen X, Mann KD, Basterfield L, Parkinson KN, Pearce MS, Reilly JK, et al. Development of sedentary behavior across childhood and adolescence: longitudinal analysis of the Gateshead millennium study. Int J Behav Nutr Phys Act. 2016;13:88.

20. van Ekris E, Wijndaele K, Altenburg TM, Atkin AJ, Twisk J, Andersen LB, et al. Tracking of total sedentary time and sedentary patterns in youth: a pooled analysis using the International Children's Accelerometry database (ICAD). Int J Behav Nutr Phys Act. 2020;17:65.

21. Cohen J. A power primer. Psychol Bull. 1992;112(1):155-9.

22. de Onis M, Onyango AW, Borghi E, Siyam A, Nishida C, Siekmann J. Development of a WHO growth reference for school-aged children and adolescents. Bull World Health Organ. 2007;85(9):660-7.

23. Lim JS, Hwang JS, Lee JA, Kim DH, Park KD, Jeong JS, et al. Cross-calibration of multi-frequency bioelectrical impedance analysis with eight-point tactile electrodes and dual-energy X-ray absorptiometry for assessment of body composition in healthy children aged 6-18 years. Pediatr Int. 2009;51(2):263-8.

24. Gába A, Mitáš J, Jakubec L. Associations between accelerometer-measured physical activity and body fatness in school-aged children. Environ Health Prev Med. 2017;22(1):1-8.

25. Troiano RP, Berrigan D, Dodd KW, Mâsse LC, Tilert T, McDowell M. Physical activity in the United States measured by accelerometer. Med Sci Sports Exerc. 2008;40(1):181-8.

26. Aadland E, Andersen LB, Anderssen SA, Resaland GK. A comparison of 10 accelerometer non-wear time criteria and logbooks in children. BMC Public Health. 2018;18:323.

27. Evenson KR, Catellier DJ, Gill K, Ondrak KS, McMurray RG. Calibration of two objective measures of physical activity for children. J Sports Sci. 2008;26(14): 1557-65.

28. Trost SG, Loprinzi PD, Moore R, Pfeiffer KA. Comparison of accelerometer cut points for predicting activity intensity in youth. Med Sci Sports Exerc. 2011;43(7):1360-8.

29. Faroog A, Martin A, Janssen X, Wilson MG, Gibson A-M, Hughes A, et al. Longitudinal changes in moderate-to-vigorous-intensity physical activity in children and adolescents: a systematic review and meta-analysis. Obes Rev 2020;21(1):e12953.

30. Altenburg TM, Chinapaw MJM. Bouts and breaks in children's sedentary time: currently used operational definitions and recommendations for future research. Prev Med. 2015;77:1-3.

31. Trost SG, Pate RR, Freedson PS, Sallis JF, Taylor WC. Using objective physical activity measures with youth: how many days of monitoring are needed? Med Sci Sports Exerc. 2000;32(2):426-31.
32. Štefelová N, Dygrýn J, Hron K, Gába A, Rubín L, Palarea-Albaladejo J. Robust compositional analysis of physical activity and sedentary behaviour data. Int J Environ Res Public Health. 2018;15(10):2248.

33. Martín-Fernández J-A, Hron K, Templ M, Filzmoser P, Palarea-Albaladejo J. Bayesian-multiplicative treatment of count zeros in compositional data sets. Stat Model. 2015;15(2):134-58.

34. Pelclová J, Štefelová N, Dumuid D, Pedišić Ž, Hron K, Gába A, et al. Are longitudinal reallocations of time between movement behaviours associated with adiposity among elderly women? A compositional isotemporal substitution analysis. Int J Obes. 2020;44(4):857-64.

35. Gába A, Dygrýn J, Štefelová N, Rubín L, Hron K, Jakubec L, et al. How do short sleepers use extra waking hours? A compositional analysis of 24-h time-use patterns among children and adolescents. Int J Behav Nutr Phys Act. 2020;17:104

36. Tanaka C, Reilly JJ, Huang WY. Longitudinal changes in objectively measured sedentary behaviour and their relationship with adiposity in children and adolescents: systematic review and evidence appraisal. Obes Rev. 2014;15(10):791-803.

37. Reilly JJ. When does it all go wrong? Longitudinal studies of changes in moderate-to-vigorous-intensity physical activity across childhood and adolescence. J Exerc Sci Fit. 2016;14(1):1-6.

38. Katzmarzyk PT, Shen W, Baxter-Jones A, Bell JD, Butte NF, Demerath EW, et al. Adiposity in children and adolescents: correlates and clinical consequences of fat stored in specific body depots. Pediatr Obes. 2012;7(5): 42-61.

39. Chong KH, Parrish A-M, Cliff DP, Kemp BJ, Zhang Z, Okely AD. Changes in physical activity, sedentary behaviour and sleep across the transition from primary to secondary school: a systematic review. J Sci Med Sport. 2020; 23(5):498-505.

40. Tassitano RM, Weaver RG, Tenório MCM, Brazendale K, Beets MW. Physical activity and sedentary time of youth in structured settings: a systematic review and meta-analysis. Int J Behav Nutr Phys Act. 2020;17:160.

41. Gába A, Dygrýn J, Štefelová N, Rubín L, Hron K, Jakubec L. Replacing school and out-of-school sedentary behaviors with physical activity and its associations with adiposity in children and adolescents: a compositional isotemporal substitution analysis. Environ Health Prev Med. 2021;26:16.

42. Mitra R, Buliung RN. Exploring differences in school travel mode choice behaviour between children and youth. Transp Policy. 2015;42:4-11.

43. Kemp BJ, Cliff DP, Chong KH, Parrish A-M. Longitudinal changes in domains of physical activity during childhood and adolescence: a systematic review. J Sci Med Sport. 2019;22(6):695-701.

44. Mann KD, Howe LD, Basterfield L, Parkinson KN, Pearce MS, Reilly JK, et al. Longitudinal study of the associations between change in sedentary behavior and change in adiposity during childhood and adolescence: Gateshead millennium study. Int J Obes. 2017;41(7):1042-7.

45. Gralla MH, McDonald SM, Breneman C, Beets MW, Moore JB. Associations of objectively measured vigorous physical activity with body composition, cardiorespiratory fitness, and cardiometabolic health in youth: a review. Am J Lifestyle Med. 2019;13(1):61-97.

46. Broadney MM, Belcher BR, Berrigan DA, Brychta RJ, Tigner IL, Shareef F, et al. Effects of interrupting sedentary behavior with short bouts of moderate physical activity on glucose tolerance in children with overweight and obesity: a randomized crossover trial. Diabetes Care. 2018;41(10):2220-8.

47. Edwardson CL, Gorely T. Epoch length and its effect on physical activity intensity. Med Sci Sports Exerc. 2010;42(5):928-34.

\section{Publisher's Note}

Springer Nature remains neutral with regard to jurisdictional claims in published maps and institutional affiliations. 